Revista de Matemática: Teoría y Aplicaciones 4(1): 61-76 (1997)

\title{
ALGUNOS POTENCIALES ALEATORIOS DEL TIPO ORNSTEIN - UHLENBECK PARA EL OPERADOR DE SCHRÖDINGER
}

\author{
Santiago Cambronero V. ${ }^{1}$
}

\begin{abstract}
Resumen
Se considera el operador de Schrödinger en el círculo de perímetro 1, con ciertos potenciales aleatorios del tipo Ornstein - Uhlenbeck, con deslizamiento dependiente del tiempo. Se describe la distribución del primer valor propio periódico para ese tipo de potenciales, basándose en la medida del movimiento browniano circular.
\end{abstract}

\section{Introducción}

El operador de Schrödinger

$$
-\frac{d^{2}}{d x^{2}}+Q
$$

ha sido usado enormemente en la modelación del movimiento de partículas, o en la descripción de cristales y otros sistemas desordenados. Vamos a considerar este operador en el círculo $S^{1}: 0 \leq x<1$. En otras palabras, consideramos un potencial $Q$ de período 1 en la recta real. Se describe la distribución del primer valor propio periódico de (1), cuando $Q$ es cierto proceso estocástico del tipo Ornstein - Uhlenbeck, con deslizamiento que depende del tiempo. En este sentido se generalizan algunos de los resultados de [5]. En esta primera sección revisamos algunas nociones y resultados obtenidos en trabajos previos del autor.

\subsection{Browniano en el círculo}

Recordemos la definición de la medida $\mu_{*}$ del movimiento browniano circular. Esta es una medida en $\mathcal{C}\left(S^{1}\right)$,definida por

$$
\mu_{*}(A)=\int_{-\infty}^{\infty} P_{a a}(A) d a
$$

\footnotetext{
${ }^{1}$ InVEStigaCión REALizAdA BAJo El Proyecto No. $114-96-331$. Centro de InvestigaCiones en Matemticas Puras y Aplicadas (Cimpa), Escuela de Matemática, Universidad de Costa Rica, 2060 San Jos, Costa Rica.
} 
donde $P_{a a}$ es la medida de probabilidad en $\mathcal{C}[0,1]$, bajo la cual el proceso de coordenadas es un movimiento browniano "atado en a" (esto es, condicionado a $B_{0}=B_{1}=a$ ).

Esta medida tiene las distribuciones finito - dimensionales

$$
\mu_{*}\left[X_{t_{0}} \in d x_{0}, \ldots, X_{t_{n-1}} \in d x_{n-1}\right]=\sqrt{2 \pi} \prod_{i=1}^{n} \mathrm{p}\left(t_{i}-t_{i-1} ; x_{i-1}, x_{i}\right) d x_{0} \ldots d x_{n-1},
$$

donde $x_{n}=x_{0}, 0=t_{0}<\ldots<t_{n}=1, \mathrm{y}$

$$
\mathrm{p}(t ; x, y)=\frac{1}{\sqrt{2 \pi t}} \exp \left[-(y-x)^{2} / 2 t\right] .
$$

Formalmente escribimos

$$
d \mu_{*}(p)=\sqrt{2 \pi} \exp \left(-\frac{1}{2} \int_{0}^{1} p^{\prime}(t) d t\right) \frac{d^{\infty} p}{(2 \pi 0+)^{\infty / 2}} .
$$

Para $\phi$ integrable bajo $\mu_{*}$ tenemos

$$
E^{*}[\phi]=\int_{-\infty}^{\infty} \int \phi(\cdot+a) d P_{00} d a
$$

La medida $\mu_{*}$ induce una medida de probabilidad $P_{0}$ en el subespacio

$$
H:=\left\{p \in \mathcal{C}\left(S^{1}\right): \int_{0}^{1} p=0\right\}
$$

de la siguiente manera

$$
P_{0}(A):=\mu_{*}\left\{p \in \Omega_{*}: p-\int_{0}^{1} p \in A, 0 \leq \int_{0}^{1} p \leq 1\right\} .
$$

y se verifica fácilmente que

$$
\int \phi(p) d \mu_{*}=\int_{-\infty}^{\infty} \int_{H} \phi(\cdot+a) d P_{0} d a
$$

para $\phi$ integrable bajo $\mu_{*}$. Para los detalles de esta construcción, ver [3] o [5].

\subsection{Ruido blanco en el círculo}

Considere un movimiento browniano $\left(b_{t}\right)_{t \geq 0}$, condicionado de tal forma que $b_{1+t}=b_{t}$. Su derivada formal $Q=b^{\prime}$ tiene entonces período 1, así que la podemos considerar como una función definida en el círculo $S^{1}$. A esta derivada formal se le llama ruido blanco en el círculo. Es fácil ver que las densidades finito-dimensionales del "proceso" $Q(t)$ están dadas por

$$
\exp \left(-\frac{1}{2} \sum_{i=1}^{n}\left(x_{i}-x_{i-1}\right)^{2} h\right) \frac{d x_{0} \ldots d x_{n-1}}{(2 \pi / h)^{n / 2}} .
$$

La medida de probabilidad correspondiente se escribe

$$
d Q_{*}(q)=\exp \left(-\frac{1}{2} \int_{0}^{1} q(t) d t\right) \frac{d^{\infty} q}{(2 \pi / 0+)^{\infty / 2}} .
$$




\subsection{La versión discreta de la ecuación de Hill}

En [2] se considera la forma discreta

$$
-\Delta^{2} u_{i}+Q_{i} u_{i}=\lambda u_{i}, \text { para } i=0,1, \ldots
$$

de la ecuación de Hill

$$
-y^{\prime \prime}+Q y=\lambda y
$$

donde $u_{i}^{\prime}=n\left(u_{i+1}-u_{i}\right), \Delta^{2} u_{i}=n^{2}\left(u_{i+1}-2 u_{i}+u_{i-1}\right), Q_{i}=n \int_{i / n}^{(i+1) / n} Q$. Se demuestra que (2) posee un primer valor propio periódico $\lambda^{(n)}$, el cual converge al primer valor propio periódico $\lambda_{0}(Q)$ de $(3)$, siempre que $b=\int_{0}^{\bullet} Q$ sea Hölder-continua.

Dada una solución $\left(u_{j}\right)_{j \geq 0}$ de $(2)$, se construye el camino poligonal $u^{(n)}$ determinado por los valores $u_{j}$ en los puntos $j / n$, y similarmente el camino $\widehat{u}^{(n)}$ determinado por los valores $u_{j}^{\prime}$. Se demuestra también que, si se imponen en $\left(u_{j}\right)_{j \geq 0}$ condiciones iniciales o cuasi-periódicas, entonces $u^{(n)}$ y $\widehat{u}^{(n)}$ convergen uniformemente en $[0,1]$ a $u$ y $u^{\prime}$, donde $u$ es la solución de $(3)$, con las mismas condiciones. Además, en el caso que $\left(u_{j}\right)_{j \geq 0}$ es la solución periódica positiva correspondiente a $\lambda^{(n)}, u^{(n)}$ y $\widehat{u}^{(n)}$ convergen uniformemente en $[0,1]$ a $u$ y $u^{\prime}$, donde $u$ es la solución periódica de (3), correspondiente a $\lambda_{0}(Q)$. Si $\lambda<\lambda_{0}(Q)$, y $m>0$, existe una solución positiva $\varphi$ de (3), que satisface

$$
\varphi(x+1)=m \varphi(x), 0 \leq x \leq 1,
$$

y para $n$ grande, existe una solución $\left(\varphi_{j}\right)$ de $(2)$ tal que $\varphi_{j+n}=\varphi_{j}, j=0,1, \ldots, n$.

La sucesión $\left(p_{j}\right)$ definida por

$$
p_{j}=n \log \frac{\varphi_{j+1}}{\varphi_{j}}, \quad\left(\text { esto es, } \varphi_{j}=\varphi_{0} \exp \left(\sum_{i=0}^{j-1} p_{i} h\right)\right)
$$

satisface

$$
Q_{i}=\lambda+n^{2}\left(e^{h p_{i+1}}-2+e^{-h p_{i}}\right), i=0,1, \ldots, n-1,
$$

y converge a la función $p=\frac{\varphi^{\prime}}{\varphi}$, en el sentido poligonal descrito arriba. Note que $p$ satisface formalmente

$$
Q=\lambda+p^{\prime}+p^{2}
$$

Todo esto es válido si $Q$ es tal que $\int_{0}^{\bullet} Q$ es Hölder - continua. Si el potencial $Q$ mismo es Hölder - continuo, se tiene que $\left(Q_{i}\right)$ converge a $Q$, en el sentido poligonal, y concluimos que

$$
\Delta^{2} \varphi_{i}=Q_{i} \varphi_{i}-\lambda \varphi_{i} \rightarrow Q(x) \varphi(x)-\lambda \varphi(x)=\varphi^{\prime \prime}(x),
$$

en el sentido poligonal. De lo anterior concluimos que $\left(p_{i}\right)$ y $\left(p_{i}^{\prime}\right)$ convergen a $p$ y $p^{\prime}$ respectivamente, en el sentido poligonal. 


\subsection{El potencial ruido blanco}

En [5], se hace uso de la transformación de Ricatti (5), para obtener una relación entre la medida de ruido blanco en el conjunto $\left[\lambda_{0} \geq \lambda\right]$, y la medida de $d \mu_{*}$ en el conjunto $H^{+}=\left[\int p \geq 0\right]$. Formalmente se tiene

$$
d Q_{*}=\exp \left[-\frac{1}{2} \int_{0}^{1} Q^{2}\right] \frac{d^{\infty} Q}{(2 \pi / 0+)^{\infty / 2}}=\frac{1}{\sqrt{2 \pi}} \exp \left[-\frac{1}{2} \int_{0}^{1}\left(\lambda+p^{2}\right)^{2}\right]|J| d \mu_{*},
$$

donde el Jacobiano $|J|$ se determina por medio de la transformación discreta (4). En el caso discreto se tiene

$$
\left|J_{n}\right|=\frac{2}{h^{n}}\left|\operatorname{senh}\left(\sum_{i=0}^{n-1} p_{i} h\right)\right|
$$

lo que permite expresar $\exp \left[-\frac{1}{2} \sum Q_{i}^{2} h\right] \frac{d Q_{0} \ldots d Q_{n-1}}{(2 \pi / h)^{n / 2}}$ como

$$
\sqrt{\frac{2}{\pi}} \exp \left[-\frac{1}{8} \sum_{i=0}^{n-1}\left(p_{i+1}^{2}+p_{i}^{2}\right)^{2} h+O\left(n^{-1}\right)\right] \operatorname{senh}\left(\sum_{i=0}^{n-1} p_{i} h\right) d \mu_{n}
$$

con

$$
d \mu_{n}=\sqrt{2 \pi} \exp \left[-\frac{1}{2 h} \sum_{i=0}^{n-1}\left(p_{i+1}-p_{i}\right)^{2}\right] \frac{d p_{0} \ldots d p_{n-1}}{(2 \pi h)^{n / 2}} .
$$

En el límite se obtiene, por convergencia dominada, que

$$
\int_{\left[\lambda_{0} \geq \lambda\right]} \phi d Q_{*}=\sqrt{\frac{2}{\pi}} \int_{H^{+}} \phi \exp \left[-\frac{1}{2} \int_{0}^{1}\left(\lambda+p^{2}\right)^{2}\right] \operatorname{senh}\left(\int_{0}^{1} p\right) d \mu_{*},
$$

para $\phi$ acotada y continua, con $\hat{\phi}(p)=\phi\left(\lambda+p^{\prime}+p^{2}\right)$ y $H^{+}=\left[\int_{0}^{1} p \geq 0\right]$. En particular, la distribución $F(\lambda)=Q_{*}\left[\lambda_{0} \geq \lambda\right]$ de $\lambda_{0}$ está dada por

$$
F(\lambda)=\sqrt{\frac{2}{\pi}} \int_{H^{+}} \exp \left[-\frac{1}{2} \int_{0}^{1}\left(\lambda+p^{2}\right)^{2}\right] \operatorname{senh}\left(\int_{0}^{1} p\right) d \mu_{*} .
$$

\section{Potencial de Ornstein-Uhlenbeck}

Consideremos el proceso de Ornstein-Uhlenbeck estándar, esto es, la difusión $Q$ que satisface la ecuación diferencial estocástica

$$
d Q=d B-m Q d t
$$

donde $B$ es un movimiento browniano estándar. $Q$ está dado explícitamente por

$$
Q(t)=Q(0) e^{-m t}+e^{-m t} \int_{0}^{t} e^{m \tau} d B_{\tau}
$$


El proceso $\int_{0}^{t} e^{m \tau} d B_{\tau}$ se puede escribir como $W\left(\frac{e^{2 m t}-1}{2 m}\right)$, para algún browniano $W$ que empieza en $0^{2}$.

Tomando $Q_{0} \equiv a$, la distribución

$$
P[Q(t) \leq b]=P\left[W\left(\frac{e^{2 m t}-1}{2 m}\right) \leq b e^{m t}-a\right]
$$

se puede expresar como

$$
\left[\frac{\pi}{m}\left(e^{2 m t}-1\right)\right]^{-\frac{1}{2}} \int_{-\infty}^{b e^{m t}-a} \exp \left[-m y^{2}\left(e^{2 m t}-1\right)^{-1}\right] d y
$$

Entonces la densidad de transición de $Q$ es

$$
\mathrm{q}(t ; a, b)=\left[\frac{\pi}{m}\left(e^{2 m t}-1\right)\right]^{-\frac{1}{2}} \exp \left[-m\left(b e^{m t}-a\right)^{2}\left(e^{2 m t}-1\right)^{-1}\right] e^{m t} .
$$

Note que $\int \mathrm{q}(t ; a, a) d a=\left(1-e^{-m t}\right)^{-1}$, para $t>0$. En particular,

$$
\int \mathrm{q}(1 ; a, a) d a=\left(1-e^{-m}\right)^{-1}<\infty .
$$

Esto no sucede para el movimiento browniano.

Queremos hallar una medida de probabilidad $\hat{Q}$, bajo la cual $Q$ sea periódico. Consideremos entonces las distribuciones finito - dimensionales

$$
\hat{Q}\left[Q\left(t_{0}\right) \in d a_{0}, \ldots, Q\left(t_{n-1}\right) \in d a_{n-1}\right]=C \prod_{i=1}^{n} \mathrm{q}\left(t_{i}-t_{i-1} ; a_{i-1}, a_{i}\right) d a_{0} \ldots d a_{n-1},
$$

donde $t_{0}=0, t_{n}=1, t_{0}<t_{1}<\ldots<t_{n}$, y $a_{n}:=a_{0}$.

En particular,

$$
\hat{Q}[Q(0) \in \mathbb{R}]=C \int \mathrm{q}(1 ; a, a) d a=\left(1-e^{-m}\right)^{-1} C,
$$

y entonces la constante adecuada es $C=1-e^{-m}$.

\subsection{Construcción vía Cameron - Martin}

Para mostrar que el proceso de Ornstein - Uhlenbeck periódico tiene una versión continua, adoptaremos un enfoque diferente.

Sean $P^{o u}$ y $P^{m b}$ las medidas de probabilidad en $C[0,1]$, bajo las cuales el proceso de coordenadas $Q_{t}(\omega)=\omega(t)$ es un Ornstein-Uhlenbeck y un movimiento browniano, repectivamente. La fórmula de Camerron - Martin nos dice que

$$
d P_{a}^{o u}(Q)=Z d P_{a}^{m b}(Q),
$$

\footnotetext{
${ }^{2}$ Ver por ejemplo [9].
} 
donde

$$
Z=Z(Q)=\exp \left[-\int_{0}^{1} m Q d Q-\frac{1}{2} \int_{0}^{1} m^{2} Q^{2} d t\right] .
$$

Defina una medida de probabilidad $\hat{Q}$ en $C[0,1]$ por

$$
\begin{aligned}
\hat{E}[\phi] & =\left.\left(1-e^{-m}\right) \int\left\{\frac{\partial}{\partial b} E_{a}^{o u}[\phi(Q), Q(1) \leq b]\right\}\right|_{b=a} d a \\
& =\left(1-e^{-m}\right) \int E_{a}^{o u}[\phi(Q) \mid Q(1)=a] \mathrm{q}(1 ; a, a) d a,
\end{aligned}
$$

para toda $\phi$ acotada y medible. La medida de probabilidad $\hat{Q}$ tiene las distribuciones finito - dimensionales dadas arriba. En efecto, tomando

$$
\phi(Q)=\chi_{\left[Q\left(t_{0}\right) \in A_{0}, \ldots, Q\left(t_{n-1}\right) \in A_{n-1}\right]},
$$

con $0=t_{0}<\ldots<t_{n}=1, \hat{Q}\left[Q\left(t_{0}\right) \in A_{0}, \ldots, Q\left(t_{n-1}\right) \in A_{n-1}\right]$ se puede escribir como

$$
C \int_{A_{0}} \frac{\partial}{\partial b}\left\{\int_{-\infty}^{b}\left(\int_{A_{1} \times \ldots \times A_{n-1}} \prod_{i=1}^{n} \mathrm{q}\left(t_{i}-t_{i-1} ; a_{i-1}, a_{i}\right) d a_{1} \ldots\right) d a_{n}\right\} d a_{0},
$$

donde la derivada se toma en $b=a_{0}$, y $C=1-e^{-m}$. Claramente esto es lo mismo que

$$
\left(1-e^{-m}\right) \int_{A_{0} \times \ldots \times A_{n-1}} \prod_{i=1}^{n} \mathrm{q}\left(t_{i}-t_{i-1} ; a_{i-1}, a_{i}\right) d a_{0} \ldots d a_{n-1},
$$

con $a_{n}=a_{0}$. Esto muestra que $\hat{Q}$ es la medida de probabilidad deseada. Ahora apliquemos la fórmula de Cameron - Martin para obtener una relación entre $\hat{Q}$ y la medida del movimiento browniano circular $\mu_{*}$. Tenemos

$$
\begin{aligned}
\hat{E}[\phi] & =\left(1-e^{-m}\right) \int\left\{\frac{\partial}{\partial b} \int_{[Q(1) \leq b]} \phi(Q) d P_{a}^{o u}\right\} d a \\
& =\left(1-e^{-m}\right) \int\left\{\frac{\partial}{\partial b} \int_{[Q(1) \leq b]} \phi(Q) Z d P_{a}^{m b}\right\} d a,
\end{aligned}
$$

donde la derivada se toma en $b=a$, y $Z$ está dada por (6). Se sigue que

$$
\begin{aligned}
\hat{E}[\phi] & =\left(1-e^{-m}\right) \int E_{a}^{m b}[\phi(Q) Z \mid Q(1)=a] \frac{d a}{\sqrt{2 \pi}} \\
& =\frac{1}{\sqrt{2 \pi}}\left(1-e^{-m}\right) e^{m / 2} \int E_{a}^{m b}\left[\phi(Q) e^{-\frac{1}{2} m^{2} \int Q^{2}} \mid Q(1)=a\right] d a \\
& =\sqrt{\frac{2}{\pi}} \operatorname{senh}\left(\frac{m}{2}\right) \int E^{00}\left[\phi(a+Q) e^{-\frac{1}{2} m^{2} \int\left(a+Q_{s}\right)^{2}}\right] d a \\
& =\sqrt{\frac{2}{\pi}} \operatorname{senh}\left(\frac{m}{2}\right) \int \phi(Q) e^{-\frac{1}{2} m^{2} \int Q^{2}} d \mu_{*} .
\end{aligned}
$$


Nota: Tomando $\phi \equiv 1$, se obtiene como corolario la identidad

$$
\int e^{-\frac{1}{2} m^{2} \int_{0}^{1} Q^{2}} d \mu_{*}=\sqrt{\frac{\pi}{2}}\left[\operatorname{senh}\left(\frac{m}{2}\right)\right]^{-1} .
$$

Equivalentemente, integrando primero en $a$,

$$
E^{00}\left(\exp \left\{-\frac{1}{2} m^{2}\left[\int_{0}^{1} Q^{2}-\left(\int_{0}^{1} Q\right)^{2}\right]\right\}\right)=\frac{m}{2 \operatorname{senh}\left(\frac{m}{2}\right)} .
$$

Con respecto a la probabilidad $P_{0}$ en $H$, obtenemos

$$
E^{0}\left[\exp \left(-\frac{m^{2}}{2} \int_{0}^{1} Q^{2}\right)\right]=\frac{m}{2 \operatorname{senh}\left(\frac{m}{2}\right)} .
$$

Este argumento se puede usar en procesos más generales, como los provenientes de ecuaciones como

$$
d Q=-m(Q) d t+d b
$$

o como

$$
d Q=-m(t) Q d t+d b .
$$

El primer caso es tratado en [5]. El segundo es objeto de estudio en la sección 3 de este trabajo.

\subsection{Distribución de $\lambda_{0}(Q)$}

Considere la ecuación de Hill (3), donde el potencial $Q$ en el proceso de OrnsteinUlenbeck periódico definido arriba. Veamos a $Q$ como un elemento de $\Omega=\mathcal{C}\left(S^{1}\right)$, donde imponemos la medida de probabilidad $\hat{Q}$. Vamos a obtener la distribución del primer valor propio de (3), siguiendo el método empleado en [5]. Dicha distribución se puede obtener de una manera más simple, como lo explicaremos en la siguiente sección, pero es importante obtener el resultado de la manera directa que lo expondremos aquí.

Como observamos en la introducción, dado que $Q$ es Hölder-continua, la solución $\left(p_{i}\right)$ de $^{3}$

$$
Q_{i}=\lambda+n^{2}\left(e^{h p_{i+1}}-2+e^{-h p_{i}}\right), \quad i=0,1, \ldots, n-1
$$

construida en [2], converge a la solución $p$ de

$$
Q=\lambda+p^{\prime}+p^{2},
$$

junto con su primera derivada. En lo que sigue, podemos asumir entonces que $\left(p_{i}^{\prime}\right)$ y $\left(p_{i}\right)$ son uniformemente acotadas.

Tome $t_{i}=i h, i=0,1, \ldots, n, y$ considere la expresión que define las distribuciones finito-dimensionales inducidas por $\hat{Q}$ en esos puntos

$$
\left(1-e^{-m}\right) \prod_{i=1}^{n} \mathrm{q}\left(t_{i}-t_{i-1} ; Q_{i-1}, Q_{i}\right)
$$

\footnotetext{
${ }^{3}$ Aquí $h=1 / n$.
} 
Esta se puede escribir como

$$
\left(e^{m}-1\right)\left[\frac{\pi}{m}\left(e^{2 m h}-1\right)\right]^{-\frac{n}{2}} \prod_{i=1}^{n} \exp \left[-\frac{1}{2}\left(Q_{i} e^{m h}-Q_{i-1}\right)^{2}\left(\frac{e^{2 m h}-1}{2 m}\right)^{-1}\right]
$$

con $a_{n}=a_{0}$. Ahora note que

$$
\left[\frac{\pi}{m}\left(e^{2 m h}-1\right)\right]^{-n / 2}=(2 \pi h)^{-n / 2} e^{-m / 2}[1+O(h)]
$$

de donde

$$
\left(e^{m}-1\right)\left[\frac{\pi}{m}\left(e^{2 m h}-1\right)\right]^{-n / 2}=2 \operatorname{senh}\left(\frac{m}{2}\right)(2 \pi h)^{-n / 2}[1+O(h)] .
$$

Por otro lado,

$$
\left(\frac{e^{2 m h}-1}{2 m}\right)^{-1}=n\left(1-m h+O\left(h^{2}\right)\right) .
$$

Tomando $\lambda=0$ por el momento,

$$
Q_{i}=p_{i}^{\prime}+\frac{1}{2}\left(p_{i}^{2}+p_{i+1}^{2}\right)+O\left(h^{2}\right)
$$

luego

$$
Q_{i} e^{m h}-Q_{i-1}=p_{i}^{\prime}-p_{i-1}^{\prime}+\frac{1}{2}\left(p_{i+1}^{2}-p_{i-1}^{2}\right)+m h p_{i}^{\prime}+\frac{m h}{2}\left(p_{i}^{2}+p_{i+1}^{2}\right)+O\left(h^{2}\right) .
$$

En particular, $p_{i}^{\prime}-p_{i-1}^{\prime}=O\left(h^{\frac{1}{2}-}\right)$, y consecuentemente $\left(Q_{i} e^{m h}-Q_{i-1}\right)^{2}$ se puede escribir como

$$
\begin{aligned}
& \left(p_{i}^{\prime}-p_{i-1}^{\prime}\right)^{2}+\left(p_{i+1}+p_{i-1}\right)\left(p_{i}^{\prime 2}-p_{i-1}^{\prime}{ }^{2}\right) h+2 m h p_{i}^{\prime}\left(p_{i}^{\prime}-p_{i-1}^{\prime}\right) \\
+ & m h\left(p_{i}^{2}+p_{i+1}^{2}\right)\left(p_{i}^{\prime}-p_{i-1}^{\prime}\right)+\frac{1}{4}\left(p_{i+1}+p_{i-1}\right)^{2}\left(p_{i}^{\prime}+p_{i-1}^{\prime}\right)^{2} h^{2} \\
+ & m\left(p_{i+1}+p_{i-1}\right)\left[\left(p_{i}^{\prime}+p_{i-1}^{\prime}\right) p_{i}^{\prime}+\frac{1}{2}\left(p_{i}^{\prime}+p_{i-1}^{\prime}\right)\left(p_{i}^{2}+p_{i+1}^{2}\right)\right] h^{2} \\
+ & m^{2}{p_{i}^{\prime}}^{2} h^{2}+m^{2}\left(p_{i}^{2}+p_{i+1}^{2}\right) p_{i}^{\prime} h^{2}+\frac{m^{2}}{4}\left(p_{i}^{2}+p_{i+1}^{2}\right)^{2} h^{2}+O\left(h^{\frac{5}{2}-}\right) .
\end{aligned}
$$

Aquí usamos que

$$
p_{i+1}^{2}-p_{i-1}^{2}=\left(p_{i+1}+p_{i-1}\right)\left(p_{i}^{\prime}+p_{i-1}^{\prime}\right) h .
$$

Cuando tomamos el producto

$$
\left(Q_{i} e^{m h}-Q_{i-1}\right)^{2}\left(1-m h+O\left(h^{2}\right)\right),
$$

obtenemos el término extra

$$
-m h\left(p_{i}^{\prime}-p_{i-1}^{\prime}\right)^{2}
$$


En la suma

$$
\sum_{i=1}^{n}\left(Q_{i} e^{m h}-Q_{i-1}\right)^{2}\left(1-m h+O\left(h^{2}\right)\right),
$$

ocurren ciertas cancelaciones. Primero, al sumar por partes,

$$
\sum\left(p_{i}^{2}+p_{i+1}^{2}\right)\left(p_{i}^{\prime}-p_{i-1}^{\prime}\right)+\sum\left(p_{i+1}+p_{i-1}\right)\left(p_{i}^{\prime}+p_{i-1}^{\prime}\right) p_{i}^{\prime} h=O(h)
$$

y también

$$
2 \sum p_{i}^{\prime}\left(p_{i}^{\prime}-p_{i-1}^{\prime}\right)-\sum\left(p_{i}^{\prime}-p_{i-1}^{\prime}\right)^{2}=\sum\left(p_{i}^{\prime 2}-p_{i-1}^{\prime}{ }^{2}\right)=0 .
$$

De lo anterior se sigue que

$$
\prod_{i=1}^{n} \exp \left[-\frac{1}{2}\left(Q_{i} e^{m h}-Q_{i-1}\right)^{2}\left(\frac{e^{2 m h}-1}{2 m}\right)^{-1}\right]
$$

es igual a

$$
\exp \left[-\frac{1}{2 h} \sum_{i=1}^{n}\left(p_{i}^{\prime}-p_{i-1}^{\prime}\right)^{2}+\Theta_{n}\right]
$$

donde $\Theta_{n}$ está dada por

$$
\begin{aligned}
& -\frac{1}{2} \sum\left(p_{i+1}+p_{i-1}\right)\left(p_{i}^{\prime 2}-p_{i-1}^{\prime}{ }^{2}\right)-\frac{h}{8} \sum\left(p_{i+1}+p_{i-1}\right)^{2}\left(p_{i}^{\prime}+p_{i-1}^{\prime}\right)^{2} \\
& -\frac{m}{4} \sum\left(p_{i+1}+p_{i-1}\right)\left(p_{i}^{\prime}+p_{i-1}^{\prime}\right)\left(p_{i}^{2}+p_{i+1}^{2}\right) h-\frac{m^{2}}{2} \sum p_{i}^{\prime 2} h \\
& -\frac{m^{2}}{2} \sum\left(p_{i}^{2}+p_{i+1}^{2}\right) p_{i}^{\prime} h-\frac{m^{2}}{8} \sum\left(p_{i}^{2}+p_{i+1}^{2}\right)^{2} h+O\left(h^{\frac{1}{2}-}\right) .
\end{aligned}
$$

Ahora como

$$
-\frac{1}{2} \sum_{i=1}^{n}\left(p_{i+1}+p_{i-1}\right)\left(p_{i}^{\prime 2}-p_{i-1}^{\prime}{ }^{2}\right)=\frac{1}{2} \sum_{i=1}^{n}\left(p_{i-1}^{\prime}{ }^{2}+p_{i+1}^{\prime}{ }^{2}\right) p_{i}^{\prime} h \rightarrow \int_{0}^{1} p^{\prime 3} d t,
$$

tenemos que $\Theta_{n}$ converge a

$$
\Theta=\int_{0}^{1}\left(p^{\prime 3}-2 p^{2} p^{\prime 2}\right) d t-\frac{m^{2}}{2} \int_{0}^{1}\left(p^{\prime}+p^{2}\right)^{2} d t .
$$

Como mencionamos anteriormente, $\Theta_{n}$ se puede asumir acotada uniformemente.

En el caso $\lambda \neq 0$, se llega a

$$
\Theta=\int_{0}^{1}\left(p^{\prime 3}-2 p^{2} p^{2}\right) d t-\frac{m^{2}}{2} \int_{0}^{1}\left(\lambda+p^{\prime}+p^{2}\right)^{2} d t .
$$

Para calcular el jacobiano, recordamos que

$$
\left|\frac{\partial Q}{\partial p}\right|=\frac{2}{h^{n}} \operatorname{senh}\left(\sum_{i=0}^{n-1} p_{i} h\right) .
$$


Tome $p_{0}=\alpha$, de modo que $p_{i}=\alpha+\left(p_{0}^{\prime}+\ldots+p_{i-1}^{\prime}\right) h$. Entonces

$$
\left|\frac{\partial p}{\partial \alpha, p^{\prime}}\right|=h^{n-1}
$$

Aquí, $p^{\prime}=\left(p_{0}^{\prime}, \ldots, p_{n-2}^{\prime}\right)$ у $p=\left(p_{0}, \ldots, p_{n-1}\right)$. Note que $p_{n}=p_{0}$ implica

$$
\sum_{i=0}^{n-1} p_{i}^{\prime} h=0
$$

Concluimos que

$$
\left|\frac{\partial Q}{\partial \alpha, p^{\prime}}\right|=\frac{2}{h} \operatorname{senh}\left(\sum_{i=0}^{n-1} p_{i} h\right)
$$

Resumiendo,

$$
\left(1-e^{-m}\right) \prod_{i=1}^{n} \mathrm{q}\left(h ; Q_{i-1}, Q_{i}\right) d Q_{0} \ldots d Q_{n-1}
$$

se transforma bajo (9) en

$$
\frac{4}{\sqrt{2 \pi}} \operatorname{senh}\left(\frac{m}{2}\right) \operatorname{senh}\left(\sum_{i=0}^{n-1} p_{i} h\right) \exp \left[\Theta_{n}\right] d \alpha d \mu_{n}
$$

con

$$
d \mu_{n}=n \sqrt{2 \pi}(2 \pi h)^{-\frac{n}{2}} \exp \left[-\frac{1}{2 h} \sum\left(p_{i}^{\prime}-p_{i-1}^{\prime}\right)^{2}\right] d \alpha d p_{0}^{\prime} \ldots d p_{n-2}^{\prime}, .
$$

En el límite obtenemos

$$
d \hat{Q}=\frac{4}{\sqrt{2 \pi}} \operatorname{senh}\left(\frac{m}{2}\right) \operatorname{senh}\left(\int_{0}^{1} p\right) \exp [\Theta] d \alpha d P_{0}\left(p^{\prime}\right),
$$

donde $P_{0}$ es la medida de probabilidad inducida por $\mu_{*}$ en $H$. Esta fórmula es válida para

$$
Q=\lambda+p^{\prime}+p^{2} \in\left[\lambda_{0} \geq \lambda\right]
$$

con $p(t)=\alpha+\int_{0}^{t} p^{\prime}, \mathrm{y}\left(\alpha, p^{\prime}\right)$ restringida a la región

$$
\left[\alpha+\int_{0}^{1} \int_{0}^{t} p^{\prime} \geq 0\right] \subset \mathbb{R} \times H .
$$

Hemos probado el siguiente resultado.

Teorema 1 Bajo la transformación $Q=\lambda+p^{\prime}+p^{2}$, la medida de probabilidad $\hat{Q}$, restringida $a\left[\lambda_{0} \geq \lambda\right]$, se relaciona con la medida $d P_{0} d \alpha$, de acuerdo con (10). 
Más precisamente, si $\phi$ es acotada y medible en $\Omega$, entonces

$$
\int_{\left[\lambda_{0} \geq \lambda\right]} \phi d \hat{Q}=C \int_{H} \int_{I\left(p^{\prime}\right)}^{\infty} \phi\left(\lambda+p^{\prime}+p^{2}\right) e^{-\frac{1}{2} m^{2} \int\left(\lambda+p^{\prime}+p^{2}\right)^{2}} G\left(\alpha, p^{\prime}\right) d \alpha d P_{0}\left(p^{\prime}\right),
$$

$\operatorname{con} C=\frac{4}{\sqrt{2 \pi}} \operatorname{senh}\left(\frac{m}{2}\right), p=\alpha+\int_{0}^{t} p^{\prime}, I\left(p^{\prime}\right)=-\int_{0}^{1} \int_{0}^{t} p^{\prime}=\int_{0}^{1} t p^{\prime}(t) d t, \mathrm{y}$

$$
G\left(\alpha, p^{\prime}\right)=\exp \left[\int_{0}^{1}\left(p^{\prime 3}-2 p^{2} p^{\prime 2}\right) d t\right] \operatorname{senh}\left(\int_{0}^{1} p\right) .
$$

Corolario 1 La distribución de $\lambda_{0}$ bajo $\hat{Q}$ está dada por

$$
\hat{Q}\left[\lambda_{0} \geq \lambda\right]=C \int_{H} \int_{I\left(p^{\prime}\right)}^{\infty} e^{-\frac{m^{2}}{2} \int\left(\lambda+p^{\prime}+p^{2}\right)^{2}} G\left(\alpha, p^{\prime}\right) d \alpha d P_{0}\left(p^{\prime}\right) .
$$

\section{Potenciales más generales}

Los resultados de la sección anterior serán ahora generalizados a potenciales más generales. Específicamente, consideramos el proceso de difusión $Q$ que resuelve

$$
d Q=-m(t) Q d t+d B
$$

donde $B$ es browniano bajo cierta medida de probabilidad $P$, y $m$ es una función derivable, con $m(t)>0$. Para $t \geq s, Q(t)$ está dado explícitamente por

$$
Q(t)=e^{-\int_{s}^{t} m}\left[Q(s)+\int_{s}^{t} e^{\int_{s}^{\tau} m} d B_{\tau}\right]
$$

El proceso $\int_{s}^{t} e^{\int_{s}^{\tau} m} d B_{\tau}$ se puede escribir como $W\left(\int_{s}^{t} e^{2 \int_{s}^{\tau} m} d \tau\right)$, para algún browniano $W$ que empieza en 0.

La identidad

$$
P[Q(t) \leq b \mid Q(s)=a]=P\left[W\left(\int_{s}^{t} e^{2 \int_{s}^{\tau} m} d \tau\right) \leq b e^{\int_{s}^{t} m}-a\right]
$$

implica que la densidad de transición $\mathrm{q}(s, t ; a, b)$ de $Q$ está dada por

$$
\left[2 \pi \int_{s}^{t} e^{2 \int_{s}^{\tau} m} d \tau\right]^{-1 / 2} \exp \left[-\left(b e^{\int_{s}^{t} m}-a\right)^{2} /\left(2 \int_{s}^{t} e^{2 \int_{s}^{\tau} m} d \tau\right)\right] e^{\int_{s}^{t} m} .
$$

Note que

$$
\int q(s, t ; a, a) d a=\frac{e^{\int_{s}^{t} m}}{e^{\int_{s}^{t} m}-1}=\left(1-e^{-\int_{s}^{t} m}\right)^{-1}<\infty .
$$

En particular,

$$
\int \mathrm{q}(0,1 ; a, a) d a=\left(1-e^{-\int_{0}^{1} m}\right)^{-1}<\infty
$$


Como en la sección anterior, queremos hallar una medida de probabilidad $\hat{Q}$, bajo la cual $Q$ sea periódico. Consideremos entonces las distribuciones finito - dimensionales

$$
\hat{Q}\left[Q\left(t_{0}\right) \in d a_{0}, \ldots, Q\left(t_{n-1}\right) \in d a_{n-1}\right]=C \prod_{i=1}^{n} \mathrm{q}\left(t_{i-1}, t_{i} ; a_{i-1}, a_{i}\right) d a_{0} \ldots d a_{n-1},
$$

donde $t_{0}=0, t_{n}=1, t_{0}<t_{1}<\ldots<t_{n}$, y $a_{n}:=a_{0}$.

En particular,

$$
\hat{Q}[Q(0) \in \mathbb{R}]=C \int \mathrm{q}(0,1 ; a, a) d a=\left(1-e^{-\int_{0}^{1} m}\right)^{-1} C,
$$

y entonces la constante adecuada es

$$
C=1-e^{-\int_{0}^{1} m}
$$

La medida de probabilidad $\hat{Q}$ está dada entonces por

$$
\begin{aligned}
\hat{E}[\phi] & =\left.\left(1-e^{-\int_{0}^{1} m}\right) \int\left\{\frac{\partial}{\partial b} E_{a}^{o u}[\phi(Q), Q(1) \leq b]\right\}\right|_{b=a} d a \\
& =\left(1-e^{-\int_{0}^{1} m}\right) \int E_{a}^{o u}[\phi(Q) \mid Q(1)=a] \mathrm{q}(1 ; a, a) d a,
\end{aligned}
$$

para toda $\phi$ acotada y medible. Esta medida de probabilidad tiene las distribuciones finito - dimensionales dadas arriba. En efecto, tomando

$$
\phi(Q)=\chi_{\left[Q\left(t_{0}\right) \in A_{0}, \ldots, Q\left(t_{n-1}\right) \in A_{n-1}\right]}
$$

con $0=t_{0}<\ldots<t_{n}=1, \hat{Q}\left[Q\left(t_{0}\right) \in A_{0}, \ldots, Q\left(t_{n-1}\right) \in A_{n-1}\right]$ se puede escribir como

$$
\left(1-e^{-\int_{0}^{1} m}\right) \int_{A_{0}} \frac{\partial}{\partial b}\left\{\int_{-\infty}^{b}\left(\int_{A_{1} \times \ldots \times A_{n-1}} \prod_{i=1}^{n} \mathrm{q}\left(t_{i-1}, t_{i} ; a_{i-1}, a_{i}\right) d a_{1} \ldots\right) d a_{n}\right\} d a_{0}
$$

donde la derivada se toma en $b=a_{0}$. Claramente esto es lo mismo que

$$
\left(1-e^{-\int_{0}^{1} m}\right) \int_{A_{0} \times \ldots \times A_{n-1}} \prod_{i=1}^{n} \mathrm{q}\left(t_{i-1}, t_{i} ; a_{i-1}, a_{i}\right) d a_{0} \ldots d a_{n-1},
$$

con $a_{n}=a_{0}$. Esto muestra que $\hat{Q}$ es la medida de probabilidad deseada. Ahora apliquemos la fórmula de Cameron - Martin para obtener una relación entre $\hat{Q}$ y la medida del movimiento browniano circular $\mu_{*}$. Tenemos

$$
\begin{aligned}
\hat{E}[\phi] & =\left(1-e^{-\int_{0}^{1} m}\right) \int\left\{\frac{\partial}{\partial b} \int_{[Q(1) \leq b]} \phi(Q) d P_{a}^{o u}\right\} d a \\
& =\left(1-e^{-\int_{0}^{1} m}\right) \int\left\{\frac{\partial}{\partial b} \int_{[Q(1) \leq b]} \phi(Q) Z d P_{a}^{m b}\right\} d a
\end{aligned}
$$


donde la derivada se toma en $b=a, \mathrm{y}$

$$
Z=\exp \left[-\int_{0}^{1} m(t) Q d Q-\frac{1}{2} \int_{0}^{1} m^{2}(t) Q^{2} d t\right] .
$$

Se sigue entonces que

$$
\begin{aligned}
\hat{E}[\phi] & =\left(1-e^{-\int_{0}^{1} m}\right) \int E_{a}^{m b}[\phi(Q) Z \mid Q(1)=a] \frac{d a}{\sqrt{2 \pi}} \\
& =\frac{1}{\sqrt{2 \pi}}\left(1-e^{-\int_{0}^{1} m}\right) \int E_{a a}^{m b}[\phi(Q) Z] d a .
\end{aligned}
$$

Ahora, aplicando la fórmula de Itô a la función $f(t, q)=\frac{1}{2} m(t) q^{2}$ tenemos

$$
\left.m(t) Q(t)\right|_{0} ^{1}=\frac{1}{2} \int_{0}^{1}\left(m^{\prime}(t) q^{2}+m(t)\right) d t+\int_{0}^{1} m(t) Q(t) d Q(t)
$$

donde $Q$ es browniano estándar. Al condicionar a $Q(1)=Q(0)=a$, y asumiendo que $m(1)=m(0)$, tenemos

$$
-\int_{0}^{1} m(t) Q(t) d Q(t)=\frac{1}{2} \int_{0}^{1}\left(m^{\prime}(t) q^{2}+m(t)\right) d t .
$$

Se sigue entonces que, bajo la medida de probabilidad $P_{a a}^{m b}$,

$$
Z=\exp \left[-\frac{1}{2} \int_{0}^{1} F(s, Q(s)) d s\right] e^{\int_{0}^{1} m}
$$

donde

$$
F(s, q)=\left[m^{2}(s)-m^{\prime}(s)\right] q^{2} .
$$

Luego,

$$
\begin{aligned}
\hat{E}[\phi] & =\frac{1}{\sqrt{2 \pi}}\left(1-e^{-\int_{0}^{1} m}\right) e^{\int_{0}^{1} m} \int E_{a a}^{m b}\left[\phi(Q) e^{-\frac{1}{2} \int_{0}^{1} F(s, Q) d s}\right] d a \\
& =\sqrt{\frac{2}{\pi}} \operatorname{senh}\left(\frac{1}{2} \int_{0}^{1} m\right) \int \phi(Q) e^{-\frac{1}{2} \int_{0}^{1} F(s, Q) d s} d \mu_{*} .
\end{aligned}
$$

Nota: Tomando $\phi \equiv 1$, se obtiene como corolario la igualdad

$$
\int e^{-\int_{0}^{1} F(s, Q) d s} d \mu_{*}=\sqrt{\frac{\pi}{2}}\left[\operatorname{senh}\left(\frac{1}{2} \int_{0}^{1} m\right)\right]^{-1} .
$$

Equivalentemente, integrando primero en $a$, y recordando que $\int_{0}^{1} m^{\prime}(t) d t=0$, obtenemos que el valor numérico de la integral

$$
E_{00}^{m b}\left(\exp \left\{-\frac{1}{2} \int_{0}^{1}\left(m^{2}-m^{\prime}\right) Q^{2}+\frac{1}{2}\left(\int_{0}^{1} m^{2}\right)^{-1}\left(\int_{0}^{1}\left(m^{2}-m^{\prime}\right) Q\right)^{2}\right\}\right)
$$


está dado por

$$
\frac{1}{2}\left(\int_{0}^{1} m^{2}\right)^{1 / 2}\left[\operatorname{senh}\left(\frac{1}{2} \int_{0}^{1} m\right)\right]^{-1} .
$$

De la misma forma tenemos que

$$
\int_{H} \exp \left\{-\frac{1}{2} \int_{0}^{1}\left(m^{2}-m^{\prime}\right) Q^{2}+\frac{1}{2}\left(\int_{0}^{1} m^{2}\right)^{-1}\left(\int_{0}^{1}\left(m^{2}-m^{\prime}\right) Q\right)^{2}\right\} d P_{0}(Q)
$$

es igual a

$$
\frac{1}{2}\left(\int_{0}^{1} m^{2}\right)^{1 / 2}\left[\operatorname{senh}\left(\frac{1}{2} \int_{0}^{1} m\right)\right]^{-1},
$$

donde $P_{0}$ es la medida de probabilidad inducida por $d \mu_{*}$ en $H=\left[\int_{0}^{1} p=0\right]$.

Hemos demostrado en particular que

$$
d \hat{Q}=\sqrt{\frac{2}{\pi}} \operatorname{senh}\left(\frac{1}{2} \int_{0}^{1} m\right) \exp \left[-\frac{1}{2} \int_{0}^{1} F(s, Q) d s\right] d \mu_{*}
$$

\subsection{Distribución de $\lambda_{0}(Q)$}

Considere la ecuación de Hill 3, donde el potencial $Q$ es el proceso de OrnsteinUlenbeck periódico generalizado, definido arriba. Veamos a $Q$ como un elemento de $\Omega=$ $C\left(S^{1}\right)$, donde imponemos la medida de probabilidad $\hat{Q}$.

La relación entre $d \hat{Q}$ y $d \mu_{*}$ obtenida arriba, nos permite reducir los cálculos al caso de un movimiento browniano circular, y luego multiplicar por el factor

$$
\sqrt{\frac{2}{\pi}} \operatorname{senh}\left(\frac{1}{2} \int_{0}^{1} m\right) \exp \left[-\frac{1}{2} \int_{0}^{1} F(s, Q) d s\right] .
$$

Ahora, los cálculos para $d \mu_{*}$ son más fáciles que en el caso de un proceso de Ornstein Uhlenbeck, descrito en la sección 2. Consideramos la versión discreta de la transformación de Ricatti

$$
Q_{i}=\lambda+n^{2}\left(e^{h p_{i+1}}-2+e^{-h p_{i}}\right), \quad i=0,1, \ldots, n-1,
$$

construida en [5].

Tome $t_{i}=i h, i=0,1, \ldots, n$, y considere la expresión que define las distribuciones finito-dimensionales inducidas por $d \mu_{*}$ en esos puntos

$$
\prod_{i=1}^{n} \mathrm{p}\left(t_{i}-t_{i-1} ; Q_{i-1}, Q_{i}\right)
$$

Esta se puede escribir como

$$
[2 \pi h]^{-n / 2} \prod_{i=1}^{n} \exp \left[-\frac{1}{2 h}\left(Q_{i}-Q_{i-1}\right)^{2}\right]
$$


$\operatorname{con} a_{n}=a_{0}$. Ahora,

$$
Q_{i}=\lambda+p_{i}^{\prime}+\frac{1}{2}\left(p_{i}^{2}+p_{i+1}^{2}\right)+O\left(h^{2}\right)
$$

luego

$$
Q_{i}-Q_{i-1}=p_{i}^{\prime}-p_{i-1}^{\prime}+\frac{1}{2}\left(p_{i+1}^{2}-p_{i-1}^{2}\right)+O\left(h^{2}\right) .
$$

En particular, $p_{i}^{\prime}-p_{i-1}^{\prime}=O\left(h^{\frac{1}{2}-}\right)$, y consecuentemente $\left(Q_{i}-Q_{i-1}\right)^{2}$ se puede escribir como

$$
\left(p_{i}^{\prime}-p_{i-1}^{\prime}\right)^{2}+\left(p_{i+1}+p_{i-1}\right)\left(p_{i}^{\prime 2}-p_{i-1}^{\prime}\right) h+\frac{1}{4}\left(p_{i+1}+p_{i-1}\right)^{2}\left(p_{i}^{\prime}+p_{i-1}^{\prime}\right)^{2} h^{2}+O\left(h^{\frac{5}{2}-}\right) .
$$

Aquí usamos que

$$
p_{i+1}^{2}-p_{i-1}^{2}=\left(p_{i+1}+p_{i-1}\right)\left(p_{i}^{\prime}+p_{i-1}^{\prime}\right) h .
$$

De lo anterior se sigue que

$$
\prod_{i=1}^{n} \exp \left[-\frac{1}{2 h}\left(Q_{i}-Q_{i-1}\right)^{2}\right]
$$

se puede escribir como

$$
\exp \left[-\frac{1}{2 h} \sum_{i=1}^{n}\left(p_{i}^{\prime}-p_{i-1}^{\prime}\right)^{2}+\Theta_{n}\right]
$$

donde $\Theta_{n}$ está dada por

$$
-\frac{1}{2} \sum\left(p_{i+1}+p_{i-1}\right)\left({p_{i}^{\prime}}^{2}-{p_{i-1}^{\prime}}^{2}\right)-\frac{h}{8} \sum\left(p_{i+1}+p_{i-1}\right)^{2}\left(p_{i}^{\prime}+p_{i-1}^{\prime}\right)^{2}+O\left(h^{\frac{1}{2}-}\right)
$$

Ahora como

$$
-\frac{1}{2} \sum_{i=1}^{n}\left(p_{i+1}+p_{i-1}\right)\left(p_{i}^{\prime 2}-p_{i-1}^{\prime}{ }^{2}\right)=\frac{1}{2} \sum_{i=1}^{n}\left(p_{i-1}^{\prime}{ }^{2}+p_{i+1}^{\prime}{ }^{2}\right) p_{i}^{\prime} h \rightarrow \int_{0}^{1} p^{\prime 3} d t
$$

tenemos que $\Theta_{n}$ converge a

$$
\Theta=\int_{0}^{1}\left(p^{\prime 3}-2 p^{2} p^{\prime 2}\right) d t
$$

Como en la sección anterior, $\Theta_{n}$ se puede asumir acotada uniformemente.

El jacobiano es el mismo que en el caso del Ornstein - Uhlenbeck,

$$
\left|\frac{\partial Q}{\partial \alpha, p^{\prime}}\right|=\frac{2}{h} \operatorname{senh}\left(\sum_{i=0}^{n-1} p_{i} h\right)
$$

En el límite obtenemos

$$
d \mu_{*}(Q)=2 \operatorname{senh}\left(\int_{0}^{1} p\right) \exp \left[\int_{0}^{1}\left(p^{\prime 3}-2 p^{2} p^{2}\right) d t\right] d \alpha d P_{0}\left(p^{\prime}\right),
$$


para

$$
Q=\lambda+p^{\prime}+p^{2} \in\left[\lambda_{0} \geq \lambda\right]
$$

con $p(t)=\alpha+\int_{0}^{t} p^{\prime}, \mathrm{y}\left(\alpha, p^{\prime}\right)$ restringida a la región $\left[\alpha+\int_{0}^{1} \int_{0}^{t} p^{\prime} \geq 0\right] \subset \mathbb{R} \times H$.

$\mathrm{Al}$ combinar este resultado con la fórmula de Cameron - Martin obtenida arriba obtenemos:

Teorema 2 Bajo la transformación $Q=\lambda+p^{\prime}+p^{2}$, la medida de probabilidad $\hat{Q}$, restringida a $\left[\lambda_{0} \geq \lambda\right]$, se relaciona con la medida $d P_{0} d \alpha$, de la siguiente manera:

$$
d \hat{Q}=C \exp \left[-\frac{1}{2} \int_{0}^{1} F\left(s, \lambda+p^{\prime}+p^{2}\right) d s\right] G\left(\alpha, p^{\prime}\right) d \alpha d P_{0}\left(p^{\prime}\right)
$$

donde $G\left(\alpha, p^{\prime}\right)$ está dada por (11), y

$$
C=2 \sqrt{\frac{2}{\pi}} \operatorname{senh}\left(\frac{1}{2} \int_{0}^{1} m\right)=\frac{4}{\sqrt{2 \pi}} \operatorname{senh}\left(\frac{1}{2} \int_{0}^{1} m\right) .
$$

Más precisamente, si $\phi$ es acotada y medible en $\Omega$, entonces

$$
\int_{\left[\lambda_{0} \geq \lambda\right]} \phi(Q) d \hat{Q}
$$

es igual a

$$
C \int_{H} \int_{I\left(p^{\prime}\right)}^{\infty} \phi\left(\lambda+p^{\prime}+p^{2}\right) \exp \left[-\frac{1}{2} \int_{0}^{1} F\left(s, \lambda+p^{\prime}+p^{2}\right) d s\right] \quad G\left(\alpha, p^{\prime}\right) d \alpha d P_{0}\left(p^{\prime}\right),
$$

$\operatorname{con} p=\alpha+\int_{0}^{t} p^{\prime}, I\left(p^{\prime}\right)=-\int_{0}^{1} \int_{0}^{t} p^{\prime}=\int_{0}^{1} t p^{\prime}(t) d t$.

Corolario 2 La distribución de $\lambda_{0}$ bajo $\hat{Q}$ está dada por

$$
\hat{Q}\left[\lambda_{0} \geq \lambda\right]=C \int_{H} \int_{I\left(p^{\prime}\right)}^{\infty} \exp \left[-\frac{1}{2} \int_{0}^{1} F\left(s, \lambda+p^{\prime}+p^{2}\right) d s\right] G\left(\alpha, p^{\prime}\right) d \alpha d P_{0}\left(p^{\prime}\right) .
$$

Note el caso particular $m(t) \equiv m>0$, donce $C=\frac{4}{\sqrt{2 \pi}} \operatorname{senh}\left(\frac{m}{2}\right)$, y se obtiene el resultado de la sección anterior.

\section{Referencias}

[1] Cambronero, S. (1996) The Distribution of the Ground State of Hill's Equation with Random Potential. Tesis para optar al Título de Ph.D. en Matemática, Instituto Courant de Ciencias Matemáticas, New York University.

[2] Cambronero, S. (1996) "La ecuación de Hill con potencial irregular", Revista de Matemática: Teoría y Aplicaciones 3(1): 25-34.

[3] Cambronero, S. (1997) "La ecuación de Hill y el movimiento browniano circular", Memorias del $V$ Encuentro Centroamericano de Investigadores en Matemática, J. Trejos (ed.), Liberia: 133-140. 
[4] Cambronero, S. (1997) "Introducción a la teoría del movimiento mrowniano", Minicurso, Memorias del $V$ Encuentro Centroamericano de Investigadores en Matemática, J. Trejos (ed.), Liberia: 33-44.

[5] Cambronero, S.; McKean, H. "On the ground state of Hill's equation with random potential", Documento en preparación para publicación.

[6] Cameron, R.H.; Martin, W.T. (1944) "Transformations of Wiener integrals under translations", $A n$ nals of Mathematics 45, No. 2.

[7] Fukushima, M.; Nakao, S. (1977) "On spectra of the Schrödinger operator with a white noise potential", Z. Wahrsch. Verb. Geb. 37: 267-274.

[8] Halperin, B.I. (1965) "Green's functions for a particle in a one-dimensional potential", Phys. Review 139, No. 1A: 104-117.

[9] Karatzas, I.; Shreve, S.E. (1991) Brownian Motion and Stochastic Calculus. Springer-Verlag. 2nd ed., New York.

[10] Lifshits, I.M.; Gradeskul, S.A.; Pastur, L.A. (1988) Introduction to the Theory of Disordered Systems. John Wiley \& Sons, New York.

[11] McKean, H.P. (1969) Stochastic Integrals. Academic Press, New York.

[12] McKean, H.P. (1994) "A limit law for the ground gtate of Hill's equation", J. of Stat. Phys. 74, Nos. 5/6: 1227-1232.

[13] McKean, H.; Cambronero, S. (1995) "Valores Propios de Dirichlet Asociados a la Ecuación de Hill con Potencial de Ruido Blanco", Revista de Matemática: Teoría y Aplicaciones 2(2): 1-7. 